\title{
Ansiedad, depresión, afecto negativo y positivo asociados con el consumo de tabaco en universitarias
}

\author{
Jennifer Lira-Mandujano', Eréndira Valdez- Piña', Mariana Núñez-Lauriano', Daniel Pech-Puebla', \\ Silvia Morales-Chainé', Sara E. Cruz Morales' \\ I Facultad de Estudios Superiores Iztacala, Universidad Nacional Autónoma de México \\ 2 Facultad de Psicología, Universidad Nacional Autónoma de México
}

\section{RESUMEN}

Introducción: en los últimos años, la prevalencia del consumo de tabaco en mujeres se ha incrementado de manera significativa a nivel nacional e internacional. Esto genera altos costos a nivel económico, familiar y de salud. Un sector de la población en el que resulta particularmente indispensable realizar investigación sobre los factores asociados con el consumo de tabaco es el de mujeres universitarias, a fin de incidir en el desarrollo de estrategias de prevención y en las intervenciones para dejar de fumar. Objetivo: determinar la asociación, entre el consumo de tabaco, la dependencia de la nicotina y enfermedades como ansiedad, depresión y afecto negativo y positivo en universitarias. Método: 72 universitarias fumadoras que asistieron de manera voluntaria a solicitar tratamiento para dejar de fumar al Centro de Atención y Orientación para Estudiantes (CAOPE) de la Facultad de Estudios Superiores Iztacala, de la Universidad Autónoma de México; se aplicaron diferentes instrumentos para conocer el patrón de consumo, el nivel de dependencia, la sintomatología depresiva y de ansiedad, y los afectos positivo y negativo. Resultados: todos los factores emocionales (ansiedad, depresión, afectos positivo y negativo) integrados en un modelo son predictores, tanto del patrón del consumo como del nivel de dependencia en universitarias; la ansiedad, en específico, aumenta el riesgo de consumo de tabaco y de presentar dependencia de la nicotina. Discusión y conclusiones: dichos resultados se deben considerar para diseñar estrategias de prevención y tratamiento para dejar de fumar en mujeres universitarias, en las que se incluyan técnicas cognitivo-conductuales. En cuanto a la prevención, es indispensable incluir estrategias enfocadas al autocontrol de emociones, a la solución de problemas y al afrontamiento para el manejo de la ansiedad.

\begin{abstract}
Introduction: In recent years the prevalence of tobacco consumption in women has increased significantly, which results in high costs at economic, family and health. Particularly, university women is a sector of the population in which is essential to carry out research on the factors associated with tobacco consumption, in order to develop prevention and intervention strategies to stop smoking. Objective: determine the association between tobacco consumption and nicotine dependence with anxiety, depression and negative and positive affect in female university students. Method: 72 female university smokers who voluntarily attended to a smoking cessation treatment in the Center of Attention and Orientation for Students (CAOPE) of the Iztacala School of Higher Studies, UNAM. Different instruments were applied to assess the pattern of consumption, the levels of dependence, depressive and anxiety symptoms, and positive and negative affect levels. Results: all emotional factors (anxiety, depression, positive and negative affect) incorporated in a model, adequately predicted both the consumption pattern and the level of dependence. In female university smokers, anxiety increased the risk of tobacco consumption and nicotine dependence. Discussion and conclusions: these results should be considered when designing prevention and treatment strategies to stop smoking in female university students, including cognitive-behavioral strategies. It is recommended to consider strategies for emotional self-control, problem solution and anxiety coping when working with smoking prevention.
\end{abstract}

Keywords: tobacco consumption, depression, anxiety, women, negative affect.

Palabras clave: consumo de tabaco, depresión, ansiedad, mujeres, afecto negativo.

Autor de correspondencia:

Daniel Pech Puebla. Carrera de Psicología. Avenida de los Barrios núm. 1, col. Los Reyes Iztacala, C.P. 54090, Tlalnepantla, Estado de México, México. Correo electrónico: daniel_070288@comunidad.unam.mx

Recibido: 22 de marzo de 2018

Aceptado: 8 de mayo de 2018

doi: 10.28931/riiad.2018.1.02 


\section{INTRODUCCIÓN}

El consumo de tabaco se considera un problema de salud pública a nivel internacional, así como la primera causa prevenible de diferentes padecimientos crónicos de alto riesgo de mortalidad, como distintos tipos de cáncer y enfermedades cardiovasculares y respiratorias, incluida la Enfermedad Pulmonar Obstructiva Crónica (EPOC; Organización Mundial de la Salud [OMS], 2017). De acuerdo con la OMS (2013), 21\% de los adultos en todo el mundo fuman (950 millones de hombres y 177 millones de mujeres). Como consecuencia, a nivel internacional, el consumo de tabaco es responsable de la muerte de más de 7 millones de personas cada año (OMS, 2017). En México, la Encuesta Nacional de Consumo de Drogas, Alcohol y Tabaco (ENCODAT, 20162017), realizada en población de 12 a 65 años, indica que existen 14.9 millones (17.6\%) de fumadores activos (11 millones 78 mil hombres y 3 millones 812 mil mujeres); $6.4 \%$ señaló fumar diariamente un promedio de 7.4 cigarros; la edad de inicio de consumo diario es de 21.4 años, en las mujeres, y de 18.8 años, en los hombres (Reynales-Shigematsu et al., 2017).

Lo anterior se ve reflejado en los costos atribuibles al uso de tabaco: 1) en México, a diario mueren 118 personas por enfermedades relacionadas con el tabaco (43 mil 246 por año); 2) anualmente, los costos de atención médica asociados con los problemas de salud provocados por el tabaco son de 61 mil 252 millones de pesos, sin embargo, dichos gastos se pueden prevenir (Pichon-Rivier et al., 2013); y 3) se ha registrado una disminución de la expectativa y la calidad de vida por fumar, expresados en Años de Vida Ajustados por la Calidad (AVAC), en mujeres es de 5.63 años y en hombres de 5.08 años. Estos datos resultan alarmantes, pues en los últimos años, el consumo de tabaco en mujeres mexicanas ha aumentado y la edad de inicio ha disminuido; a este fenómeno se le denomina "feminización de la epidemia" (González-Roldán, 2015).

Estudios sobre las consecuencias del consumo de tabaco en la salud de las mujeres muestran que, además de tener vinculación con el cáncer y enfermedades cardiovasculares y pulmonares, fumar también se relaciona con consecuencias en la salud reproductiva, como riesgo de mayor tasa de infertilidad, síndrome de muerte súbita del lactante, cáncer de cuello uterino, ciclos de menstruación irregular, dismenorrea y menopausia temprana; en el caso de las embarazadas, se ha documentado parto prematuro, bajo peso al nacer y embarazo ectópico. Estos padecimientos impactan, además, el bienestar económico de las mujeres y sus familias (Centers for Disease Control and Prevention, Na- tional Center for Chronic Disease Prevention and Health Promotion, \& Office on Smoking and Health, 2010; Regueira, Suárez-Lugo, \& Jakimczuk, 2010; Suárez \& Campos, 2010).

A partir de diferentes estudios transversales, se sabe que los factores asociados con el inicio y la progresión del consumo de tabaco en mujeres son la alta sintomatología depresiva, la vulnerabilidad a la depresión, el bajo afecto positivo y el alto afecto negativo (Audrain-McGovern, Rodriguez, \& Leventhal, 2014; Larsen, Otten, \& Engels, 2009). En cuanto a los factores asociados con el mantenimiento del consumo de tabaco en mujeres adultas, se identifican síntomas de depresión y ansiedad, así como un alto afecto negativo (Goodwin, Pagura, Spiwak, Lemeshow, \& Sareen, 2011; Leventhal et al., 2007; Luk \& Tsoh, 2010; Nakajima \& al'Absi, 2012; Roys, Weed, Carrigan, \& MacKillop, 2016). Se sabe que la comorbilidad de ansiedad y depresión es un factor que aumenta la probabilidad del consumo de sustancias, particularmente en el caso del consumo de tabaco en mujeres (Dahne, Hoffman, \& MacPherson, 2015). La literatura científica muestra que cuando las mujeres suprimen el consumo de tabaco presentan síntomas de abstinencia más graves que los hombres, es decir que se incrementan el afecto negativo (miedo, ansiedad, tristeza, irritabilidad, tensión, impaciencia, inquietud), la angustia relacionada con la abstinencia y el deseo de fumar para aliviarla, además de los problemas de concentración y los síntomas físicos desagradables (dolores de cabeza, síntoma autonómico y somnolencia; Leventhal et al., 2007; Pang \& Leventhal, 2013; Perkins, Karelitz, Giedgowd, \& Conklin, 2013). Dado que las mujeres experimentan síntomas de abstinencia severos, utilizan el cigarro como reforzador negativo para aliviarlos (Pang, Zvolensky, Schmidt, \& Leventhal, 2015; Schleicher, Harris, Catley, \& Nazir, 2009; Weinberger \& McKee, 2012; Xu et al., 2008).

Se ha observado que después de asistir a intervenciones para dejar de fumar, las tasas de abstinencia de las mujeres son muy bajas y se presentan diferentes efectos secundarios, específicamente cuando se emplean terapias de sustitución con nicotina y terapias farmacológicas no nicotínicas (Pang et al., 2015; Schmitz, Stotts, Mooney, DeLaune, \& Moeller, 2007; Schnoll, Patterson, \& Lerman, 2007).

A pesar de la existencia de diversos estudios sobre los efectos del consumo de tabaco y los tratamientos para dejar de fumar estandarizados disponibles en México que han demostrado su efectividad, es indispensable investigar sobre los factores asociados con el consumo de tabaco en mujeres, a fin de que los resultados tengan un impacto en el desarrollo de estrategias de prevención y en las intervenciones para dejar de fumar enfocadas en universitarias. La ENCODAT (Reynales-Shigematsu et al., 
2017) señala que en la población de 18 a 24 años (que coincide con la edad en la que muchas adultas jóvenes asisten a la universidad), $23.3 \%$ de quienes afirmaron fumar consume en promedio 5.1 cigarros; además, $4.2 \%$ tiene adicción a la nicotina. La misma encuesta señala que de las mujeres de 18 a 24 que señalaron fumar, 63.5\% intentó dejar de hacerlo en los últimos 12 meses, pero $0 \%$ se sometió a un tratamiento; por el contrario, $56.2 \%$ intento dejar el tabaco súbitamente.

La universidad es un periodo transicional único, en el cual los estudiantes experimentan altos niveles de depresión y estrés por las exigencias académicas y sociales. Por lo tanto, no es sorprendente que la relación depresión-fumar se extienda a estudiantes universitarios (Schleicher et al., 2009). Morrell, Cohen y McChargue (2010) explican que un porcentaje importante de mujeres inicia la licenciatura sin fumar y finaliza la misma con consumo ocasional o diario de cigarros. En estudios epidemiológicos realizados en nuestro país, se señala que las drogas más comunes de consumo entre los estudiantes de nivel superior son principalmente tabaco y alcohol (Secretaría de Salud, 2012; Villatoro et al., 2013). Por ello, el objetivo del presente trabajo es determinar la asociación entre el uso de tabaco, la dependencia de la nicotina y enfermedades como la ansiedad, la depresión y el afecto negativo y positivo en estudiantes universitarias. Como objetivo específico se planteó evaluar la diferencia en el patrón de consumo y el nivel de dependencia, de acuerdo con los niveles de ansiedad, depresión y afectos positivo y negativo.

\section{MÉTODO}

\section{Participantes}

72 mujeres universitarias fumadoras que asistieron voluntariamente a solicitar tratamiento al Centro de Atención y Orientación para Estudiantes (CAOPE) de la Facultad de Estudios Superiores Iztacala, de la UNAM, para dejar de fumar tabaco; las participantes tenían una media de edad de 21.63 años $(D E=2.61)$, la edad más baja fue de 18 y la más alta de 32 años. La media del patrón de consumo de las participantes fue de 3.26 ( $D E$ $=2.86$ ) cigarros diarios en el mes anterior a la evaluación; quien menos consumió presentó un consumo de .20 cigarros, y quien más lo hizo, uno de 14.70 cigarros. La media de edad de inicio de consumo de las participantes fue de $15.06(D E=2.18)$ años. Con respecto al nivel de dependencia, las mujeres obtuvieron un puntaje medio de $2.81(D E=1.65)$ puntos, lo que equivale a un nivel de dependencia baja.

Criterios de inclusión: ser fumadoras interesadas en dejar de serlo, que hayan consumido a diario más de dos cigarros durante el último año y hasta un mes antes de la intervención, y que no hayan tenido un periodo de abstinencia mayor a tres meses en el último año.

Criterios de exclusión: tener historial de cáncer; consumir los siguientes medicamentos: antidepresivos, antipsicóticos, anticonvulsivos, naltrexona, esteroides o insulina; estar asistiendo a otro tratamiento para dejar de fumar o a alguna terapia psicológica; presentar dependencia de otras drogas (mariguana, cocaína, inhalables, etcétera).

\section{Instrumentos}

Entrevista inicial: tiene como objetivo recopilar datos relacionados con aspectos socio-demográficos de las participantes; está constituida por 28 preguntas relacionadas con edad, género e historia de consumo de tabaco.

Consentimiento informado: se utiliza para proporcionar información acerca de los objetivos de la investigación. También se usa para garantizar la confidencialidad de los datos, para aclarar puntos importantes de la participación en el estudio y para solicitar el consentimiento explícito de las participantes.

Línea Base Retrospectiva (LIBARE; Sobell \& Sobell, 1980): se utiliza con el fin de conocer el patrón de consumo de tabaco durante los treinta días previos al estudio. La adaptación de este instrumento para diferentes substancias, incluyendo tabaco, fue realizada por Robinson, Sobell, Sobell y Leo (2014); arrojó un nivel de confiabilidad por test-retest con correlaciones a partir de .75 y hasta .96, para las versiones de 30, 90 y 360 días de evaluación de consumo.

Cuestionario de Fageström para Dependencia de la Nicotina (TFDN; Heatherton, Kozlowski, Frecker, \& Fagerström, 1991): consta de seis reactivos en los que se evalúa la dependencia de la nicotina, a través de preguntas relacionadas con la intensidad, la frecuencia y el control de consumo.

Inventario de Depresión de Beck (IDB; Beck, Steer, \& Gabin, 1988): evalúa la sintomatología relacionada con la depresión. Consta de 21 reactivos compuestos de cuatro frases cada uno, en los que las personas evaluadas eligen la que más se adapte a la forma en que se sintieron en la semana previa a su evaluación, incluyendo el día de la aplicación. En su versión adaptada para población mexicana, este instrumento mostró una confiabilidad por alfa de Cronbach de .87 (Jurado et al., 1998).

Inventario de Ansiedad de Beck (IAB; Beck, Epstein, Brown, \& Steer, 1988): mide el nivel de sintomatología relacionada con la ansiedad, a través de 21 reactivos en los que se pide a las personas evaluadas que elijan la intensidad con la que han experimentado los síntomas 
en la última semana, incluyendo el día de la evaluación, a partir de una escala tipo Likert de cuatro opciones. La versión adaptada para población mexicana de este instrumento, realizada por Robles, Varela, Jurado y Páez (2001) cuenta con una confiabilidad por alfa de Cronbach de 83 .

Escalas de afectos positivo y negativo (PANAS; Watson, Clark, \& Tellegen, 1988): evalúa los niveles de afectos positivo y negativo que la persona experimenta en la semana previa a la aplicación y de forma general. Consta de 20 reactivos que analizan, en una escala tipo Likert, la intensidad en que se han experimentado cada uno de los estados de ánimo incluidos. Los reactivos se repiten para la sección semanal y la general. La versión adaptada para población mexicana fue realizada por Robles y Páez (2003), y obtuvo coeficientes de alfa de Cronbach de .85 a .90 para el afecto positivo, tanto semanal como general, y de .81 a .85 para el afecto negativo, tanto semanal como general.

\section{Procedimiento}

Los datos se obtuvieron de la evaluación inicial que forma parte del tratamiento para dejar de fumar. Se realizó difusión sobre el tratamiento, a fin de captar a posibles participantes; se contactó a las candidatas interesadas para agendar una sesión con un terapeuta que dominaba la aplicación del tratamiento para dejar de fumar. Posteriormente, se les explicó el objetivo del tratamiento y se les proporcionó un documento de consentimiento informado para que lo leyeran y firmaran, en caso de estar de acuerdo. Tras esto, a las que aceptaron participar en el estudio se les aplicaron los instrumentos sobre el patrón de consumo de cigarros y sus niveles de depresión, ansiedad y afecto negativo y positivo.

Los datos recopilados a partir de la aplicación de los instrumentos fueron capturados en una base de datos en el programa ${ }^{\circledR} I B M$ SPSS Statistics 21, para su posterior análisis.

\section{Análisis estadísticos}

Con el fin de conocer las características de la muestra, con respecto a las variables exploradas, se realizaron análisis de estadística descriptiva (media, desviación estándar, rango) con respecto a las puntuaciones de patrón de consumo (LIBARE), dependencia (TFDN), sintomatología de depresión (IDB), sintomatología de ansiedad (IAB) y afectos positivo y negativo, de forma semanal y general (PANAS).

Asimismo, para conocer si el patrón de consumo y el nivel de dependencia varían de acuerdo con el nivel de ansiedad, depresión y afectos positivo y negativos, se realizaron análisis de varianza de un factor (ANOVA), con el fin de saber en dónde existían diferencias; si éstas eran significativas, se realizaban pruebas post-hoc de Bonferroni.

Con el propósito de evaluar la forma en que los factores emocionales (sintomatología de depresión, ansiedad y afectos positivo y negativo) se asocian con el patrón de consumo y la dependencia, se realizó un análisis de regresión lineal múltiple.

\section{RESULTADOS}

Las características de la muestra se observan en la Tabla 1; las participantes presentaron una media de síntomas de depresión de $14.19(D E=9.43)$ puntos y de ansiedad de 17.58 ( $D E=11.07)$, lo que equivale a un nivel leve y moderado, respectivamente. Además, se observó una media de afecto positivo semanal de 28.40 ( $D E=$ 8.61) puntos, mientras que para el afecto negativo semanal la media fue de $25.81(D E=8.64)$ puntos; en afecto positivo general mostraron una media de $31.64(D E=$ 8.53) puntos y en afecto negativo general una media de $23.50(D E=7.46)$ puntos.

Tabla 1

Características de la muestra $(n=72)$

\begin{tabular}{lc}
\hline & $\bar{X}(D E)$ \\
\hline Intentos para dejar de fumar & $1.53(1.60)$ \\
Años de consumo regular & $4.60(2.68)$ \\
\hline & $n(\%)$ \\
Han usado tratamiento farmacológico & $1(1.4)$ \\
Vive con fumadores & $37(51.4)$ \\
\hline
\end{tabular}

Como se muestra en la Tabla 2, al efectuar diferentes pruebas ANOVA para evaluar las diferencias en el patrón de consumo y el puntaje de dependencia, se tomaron como grupos a los niveles de ansiedad, depresión y afectos positivo y negativo; se observó que el patrón de consumo y el puntaje de dependencia difieren entre los niveles de ansiedad $F(3)=3.17, p=.3 ; F(3)=4.06, p$ $=.1$, respectivamente). Las pruebas de Bonferroni mostraron diferencias significativas $(p=.04)$ en el puntaje de dependencia entre quienes tenían ansiedad leve ( $\bar{X}=$ 2.23, $D E=0.88)$ y quienes presentaban ansiedad moderada $(\bar{X}=3.38, D E=2.09)$, lo cual indica que quienes tienen un nivel de ansiedad moderada presentan mayor dependencia con respecto a las que tienen ansiedad leve (ver Tabla 3). 
Tabla 2

Resultados de ANOVA de un factor, con respecto al patrón de consumo y nivel de dependencia, tomando en cuenta los niveles de cada variable

\begin{tabular}{|c|c|c|c|}
\hline & Grupos & $F(g l)$ & $p$ \\
\hline \multirow[t]{6}{*}{ Patrón de consumo } & Ansiedad (mínima, leve, moderada, severa) & $3.17(3)$ & $.030^{\star}$ \\
\hline & Depresión (mínima, leve, moderada, severa) & $.441(3)$ & .725 \\
\hline & Niveles de afecto positivo semanal (bajo, moderado, alto) & $.244(2)$ & .784 \\
\hline & Niveles de afecto negativo semanal (bajo, moderado, alto) & $1.462(2)$ & .239 \\
\hline & Niveles de afecto positivo general (bajo, moderado, alto) & $.805(2)$ & .451 \\
\hline & Niveles de afecto negativo general (bajo, moderado, alto) & $.06(2)$ & .942 \\
\hline \multirow[t]{6}{*}{ Nivel de dependencia } & Ansiedad (mínima, leve, moderada, severa) & $4.06(3)$ & $.01^{*}$ \\
\hline & Depresión (mínima, leve, moderada, severa) & $.601(3)$ & .617 \\
\hline & Niveles de afecto positivo semanal (bajo, moderado, alto) & $.862(2)$ & .427 \\
\hline & Niveles de afecto negativo semanal (bajo, moderado, alto) & $.879(2)$ & .420 \\
\hline & Niveles de afecto positivo general (bajo, moderado, alto) & $1.341(2)$ & .268 \\
\hline & Niveles de afecto negativo general (bajo, moderado, alto) & $.219(2)$ & .804 \\
\hline
\end{tabular}

* Significativo al nivel $<.05$

Tabla 3

Resultados de prueba post hoc de Bonferroni de las diferencias en el patrón de consumo y el nivel de dependencia, de acuerdo con los niveles de ansiedad

\begin{tabular}{|c|c|c|c|c|c|c|}
\hline & & & & & $\begin{array}{l}95 \% \text { Inter } \\
\text { para la dif }\end{array}$ & $\begin{array}{l}\text { confianza } \\
\text { de media }\end{array}$ \\
\hline & Grupo (I) & Grupo (J) & $\begin{array}{l}\text { Diferencia de } \\
\text { medias (I-J) }\end{array}$ & $p$ & $\begin{array}{l}\text { Límite } \\
\text { inferior }\end{array}$ & $\begin{array}{l}\text { Límite } \\
\text { superior }\end{array}$ \\
\hline \multirow{6}{*}{ Patrón de consumo } & \multirow{3}{*}{$\begin{array}{l}\text { Ansiedad } \\
\text { mínima }\end{array}$} & $\begin{array}{l}\text { Ansiedad } \\
\text { leve }\end{array}$ & -.36 & 1 & -3.68 & 2.96 \\
\hline & & $\begin{array}{l}\text { Ansiedad } \\
\text { moderada }\end{array}$ & -2.14 & .535 & -5.51 & 1.23 \\
\hline & & $\begin{array}{l}\text { Ansiedad } \\
\text { severa }\end{array}$ & -2.62 & .444 & -6.54 & 1.3 \\
\hline & \multirow{2}{*}{$\begin{array}{l}\text { Ansiedad } \\
\text { leve }\end{array}$} & $\begin{array}{l}\text { Ansiedad } \\
\text { moderada }\end{array}$ & -1.77 & .103 & -3.75 & .2 \\
\hline & & $\begin{array}{l}\text { Ansiedad } \\
\text { severa }\end{array}$ & -2.26 & .197 & -5.07 & .55 \\
\hline & $\begin{array}{l}\text { Ansiedad } \\
\text { moderada }\end{array}$ & $\begin{array}{l}\text { Ansiedad } \\
\text { severa }\end{array}$ & -.48 & 1 & -3.35 & 2.39 \\
\hline \multirow{6}{*}{ Nivel de dependencia } & \multirow{3}{*}{$\begin{array}{l}\text { Ansiedad } \\
\text { mínima }\end{array}$} & $\begin{array}{l}\text { Ansiedad } \\
\text { leve }\end{array}$ & -.226 & 1 & -2.12 & 1.67 \\
\hline & & $\begin{array}{l}\text { Ansiedad } \\
\text { moderada }\end{array}$ & -1.38 & .325 & -3.31 & .54 \\
\hline & & $\begin{array}{l}\text { Ansiedad } \\
\text { severa }\end{array}$ & -1.667 & .28 & -3.9 & .57 \\
\hline & \multirow{2}{*}{$\begin{array}{l}\text { Ansiedad } \\
\text { leve }\end{array}$} & $\begin{array}{l}\text { Ansiedad } \\
\text { moderada }\end{array}$ & -1.15 & $.041^{*}$ & -2.29 & -.03 \\
\hline & & $\begin{array}{l}\text { Ansiedad } \\
\text { severa }\end{array}$ & -1.44 & .104 & -3.05 & .16 \\
\hline & $\begin{array}{l}\text { Ansiedad } \\
\text { moderada }\end{array}$ & $\begin{array}{l}\text { Ansiedad } \\
\text { severa }\end{array}$ & -.282 & 1 & -1.92 & 1.36 \\
\hline
\end{tabular}


Al analizar la asociación existente entre los factores emocionales se encontró, a través de análisis de regresiones lineales múltiples, que este modelo se asocia tanto con el patrón de consumo $(F=3.087, p=.010)$ como con el nivel de dependencia $(F=2.661, p=.023)$. Como se puede observar en la Tabla 4, los síntomas de depresión disminuyen el riesgo de consumo de tabaco $(\mathrm{B}=-0.099,95 \% \mathrm{IC}=-0.193$ a $-0.005, p=.040)$, mientras que la ansiedad la aumenta $(B=0.121,95 \% \mathrm{IC}=0.042$ a $0.200, p=.003$ ). De la misma forma y como se ve en la Tabla 5, la sintomatología de ansiedad aumenta el riesgo de presentar dependencia de la nicotina $(B=0.074$, $95 \% \mathrm{IC}=0.027$ a $0.120, p=.002$ ).

\section{DISCUSIÓN Y CONCLUSIONES}

El objetivo del presente trabajo fue determinar la asociación entre el consumo de tabaco, la dependencia de la nicotina y la ansiedad, la depresión, el afecto negativo y el positivo en universitarias. De acuerdo con los análisis de regresión realizados, se encontró que todos los factores emocionales (ansiedad, depresión, afectos positivo y negativo) logran predecir adecuadamente tanto el patrón de consumo como el nivel de dependencia; en específico, las variables de ansiedad y depresión -en especial, la primera-aportan más al modelo y logran predecir adecuadamente el patrón de consumo, lo cual demuestra que estos factores emocionales se encuentran fuertemente asociados con el patrón de consumo y el nivel de dependencia en mujeres universitarias que solicitan tratamiento para dejar de fumar.

Los resultados coinciden con diferentes estudios (Goodwin et al., 2011; Leventhal et al., 2007; Luk \& Tsoh, 2010; Nakajima \& al'Absi, 2012; Pang et al., 2015; Roys et al., 2016; Schleicher et al., 2009; Weinberger \& McKee, 2012; Xu et al., 2008) en los que se muestra una asociación significativa entre la sintomatología depresiva y el afecto negativo en mujeres adultas y el nivel de

Tabla 4

Regresión lineal múltiple de modelo de factores emocionales que predicen el patrón de consumo

\begin{tabular}{|c|c|c|c|c|c|c|c|c|}
\hline \multicolumn{5}{|c|}{ Resumen del modelo } & \multicolumn{4}{|c|}{ Aporte al modelo de cada VI } \\
\hline \multirow[b]{2}{*}{$\begin{array}{c}\text { Variables } \\
\text { independientes }\end{array}$} & \multirow[b]{2}{*}{$\begin{array}{c}\text { Variable } \\
\text { dependiente }\end{array}$} & \multirow[b]{2}{*}{$F$} & \multirow[b]{2}{*}{$R^{2}$} & \multirow[b]{2}{*}{$p$} & \multirow[b]{2}{*}{$\mathrm{B}$} & \multirow[b]{2}{*}{$p$} & \multicolumn{2}{|c|}{$95 \%$ IC para $B$} \\
\hline & & & & & & & $\begin{array}{l}\text { Límite } \\
\text { inferior }\end{array}$ & $\begin{array}{l}\text { Límite } \\
\text { superior }\end{array}$ \\
\hline Síntomas de depresión & \multirow{6}{*}{$\begin{array}{c}\text { Patrón } \\
\text { de } \\
\text { consumo }\end{array}$} & \multirow{6}{*}{3.087} & \multirow{6}{*}{$22.2 \%$} & \multirow{6}{*}{$.010^{*}$} & -.099 & $.040^{*}$ & -.193 & -.005 \\
\hline Síntomas de ansiedad & & & & & .121 & $.003^{*}$ & .042 & .200 \\
\hline Afecto positivo semanal & & & & & .057 & .268 & -.045 & .159 \\
\hline Afecto negativo semanal & & & & & .086 & .108 & -.019 & .191 \\
\hline Afecto positivo general & & & & & -.102 & .039 & -.199 & -.005 \\
\hline Afecto negativo general & & & & & -.078 & .151 & -.185 & .029 \\
\hline
\end{tabular}

* Significativo al nivel $p \leq .05$

Tabla 5

Regresión lineal múltiple de modelo de factores emocionales que predicen el nivel de dependencia

\begin{tabular}{|c|c|c|c|c|c|c|c|c|}
\hline \multicolumn{5}{|c|}{ Resumen del modelo } & \multicolumn{4}{|c|}{ Aporte al modelo de cada VI } \\
\hline \multirow[b]{2}{*}{$\begin{array}{c}\text { Variables } \\
\text { independientes }\end{array}$} & \multirow[b]{2}{*}{$\begin{array}{c}\text { Variable } \\
\text { dependiente }\end{array}$} & \multirow[b]{2}{*}{$F$} & \multirow[b]{2}{*}{$R^{2}$} & \multirow[b]{2}{*}{$p$} & \multirow[b]{2}{*}{$\mathrm{B}$} & \multirow[b]{2}{*}{$p$} & \multicolumn{2}{|c|}{$95 \%$ IC para B } \\
\hline & & & & & & & $\begin{array}{l}\text { Límite } \\
\text { inferior }\end{array}$ & $\begin{array}{l}\text { Límite } \\
\text { superior }\end{array}$ \\
\hline Síntomas de depresión & \multirow{6}{*}{$\begin{array}{c}\text { Nivel } \\
\text { de } \\
\text { dependencia }\end{array}$} & \multirow{6}{*}{2.661} & \multirow{6}{*}{$19.7 \%$} & \multirow{6}{*}{$.023^{*}$} & -.022 & .425 & -.078 & .033 \\
\hline Síntomas de ansiedad & & & & & .074 & $.002^{*}$ & .027 & .120 \\
\hline Afecto positivo semanal & & & & & .031 & .304 & -.029 & .091 \\
\hline Afecto negativo semanal & & & & & .010 & .754 & -.052 & .071 \\
\hline Afecto positivo general & & & & & -.048 & .095 & -.106 & .009 \\
\hline Afecto negativo general & & & & & -.014 & .650 & -.078 & .049 \\
\hline
\end{tabular}

* Significativo al nivel $p \leq .05$ 
dependencia. Sin embargo, los resultados obtenidos en el presente estudio incluyen la ansiedad como una variable que también se asocia con el patrón de consumo y al nivel de dependencia de la nicotina.

Por lo tanto, la información obtenida en el presente estudio resulta de gran importancia en la instrumentación de estrategias de prevención del consumo de tabaco y en el diseño de tratamientos para dejar de fumar dirigidos a mujeres universitarias, ya que apoyan la propuesta planteada tanto por la Oficina de las Naciones Unidas contra la Droga y el Delito (UNODC, 2016) como por la OMS (2010), de diseñar estrategias de prevención y tratamiento del consumo de drogas que estén enfocadas en la mujeres, de acuerdo con las características de cada etapa de su desarrollo (niñez, adolescencia, juventud y adultez). A partir de los resultados obtenidos en el presente estudio, se sugiere para futuras investigaciones diseñar y evaluar el impacto de una intervención para dejar de fumar dirigida a mujeres universitarias, que incluya estrategias cognitivo- conductuales que cuenten con evidencia enfocada al manejo de la ansiedad y los síntomas de depresión; se recomienda, en específico, incluir técnicas de autocontrol y de control de la activación, así como habilidades en solución de problemas, entrenamiento en autoinstrucciones y regulación emocional.

De la misma forma, se propone realizar con niñas un estudio en el que se evalúe el impacto de la aplicación de estrategias de prevención enfocadas al autocontrol de emociones, a la solución de problemas y al afrontamiento para el manejo de la ansiedad, en el contexto de un estudio longitudinal.

Adicionalmente, una variable que debe incluirse en futuras investigaciones es el estrés. En una revisión realizada por Torres y O'Dell (2016) se señala que las mujeres tienen mayor predisposición al estrés y a la ansiedad, y que al experimentarlos son más susceptibles a fumar para hacerles frente.

El estrés es un factor que se ha relacionado con el inicio y el mantenimiento del consumo de tabaco (Wood, Cano-Vindel, Iruarrizaga, Dongil, \& Salguero, 2010). Sin embargo, poco se sabe sobre la relación entre este factor y el uso de tabaco en mujeres universitarias; es importante conocer dicho aspecto, pues algunos autores señalan que el ingreso a la universidad genera estrés por la necesidad de adaptarse a un nuevo ambiente y a nuevas demandas (Toribio-Ferrer \& Franco-Bárcenas, 2016). Por lo tanto, identificar los factores que generan estrés podría ayudar en la instrumentación de tratamientos efectivos para la reducción de los niveles de estrés en población universitaria (Pozos-Radillo, Preciado-Serrano, Acosta-Fernández, Aguilera-Velasco, \& Delgado-García, 2014; Pozos-Radillo, Preciado-Serrano,
Plascencia, Acosta-Fernández, \& Aguilera, 2015; Jerez-Mendoza \& Oyarzo-Barría, 2015), así como en la instrumentación de estrategias de prevención y tratamientos para dejar de fumar en este sector.

\section{FUENTES DE FINANCIAMIENTO}

El presente trabajo se realizó gracias al Programa de Apoyo a Proyectos de Investigación e Innovación Tecnológica (PAPIIT) de la Dirección General de Asuntos del Personal Académico (DGAPA- UNAM). PAPIIT -IA304016 RA304016.

\section{CONFLICTOS DE INTERÉS}

No existen conflictos de interés.

\section{REFERENCIAS}

Audrain-McGovern, J., Rodriguez, D., \& Leventhal, A. M. (2014). Gender differences in the relationship between affect and adolescent smoking uptake. Addiction, 110(3), 519-529. doi: 10.1111/ add. 12797

Beck, A. T., Epstein, N., Brown, G., \& Steer, R. A. (1988). An inventory for measuring clinical anxiety: psychometric properties. Journal of Consulting and Clinical Psychology, 56(6), 893-897.

Beck, A. T., Steer, R. A., \& Gabin, M. G. (1988). Psychometric properties of the beck depression inventory: Twenty-five years of evaluation. Clinical Psychology Review, 8(1), 77-100.

Centers for Disease Control and Prevention, National Center for Chronic Disease Prevention and Health Promotion, \& Office on Smoking and Health. (2010). How tobacco smoke causes disease. The biology and behavioral basis for smoking-attributable disease: a report of the surgeon general. Atlanta: Publications and Reports of the Surgeon General. Recuperado de: https:// www.ncbi.nlm.nih.gov/pubmed/21452462

Dahne, J., Hoffman, E. M., \& MacPherson, L. (2015). The association between anxiety sensivity and motivation to quit smoking among women and men in residential substance use treatment. Substance Use \& Misuse, 50(1), 72-78. doi: 10.3109/10826084.2014.958856

González-Roldán, F. J. (2015). Tabaco: avances y necesidades de información. En M. E., Medina-Mora (Coord.). Hacia dónde va la ciencia en México. Salud Mental y Adicciones. México: CONAСуT, Academia Mexicana de Ciencias y Consejo Consultivo de Ciencias Presidencia de la República.

Goodwin, R., Pagura, J., Spiwak, R., Lemeshow, A., \& Sareen, J. (2011). Predictors of persistent nicotine dependence among adults in the United States. Drug Alcohol Dependence, 118(2), 127-133. doi:10.1016/j.drugalcdep.2011.03.010

Heatherton, T. F., Kozlowski, L. T., Frecker, R. C., \& Fagerström, K. O. (1991). The Fagerström Test for Nicotine Dependence: a revi- 
sion of the Fagerström Tolerance Questionnaire. British Journal of Addiction to Alcohol and other Drugs, 86(9), 1119-1127.

Jerez-Mendoza, M., \& Oyarzo-Barría, C. (2015). Estrés académico en estudiantes del departamento de salud de la Universidad de Los Lagos Osorno. Revista Chilena de Neuro-Psiquiatría, 53(3), 149-157.

Jurado, S., Villegas, M., Méndez, L., Rodríguez, F., Loropena, V., \& Varela, R. (1998). La estandarización del inventario de depresión de Beck para los residentes de la Ciudad de México. Salud Mental, 21(3), 26-31.

Larsen, J. K., Otten, R., \& Engels, R. (2009). Adolescent depressive symptoms and smoking behavior: the gender-specific role of weight concern and dieting. Journal of Psychosomatic Research, 66(4), 305-308. doi: 10.1016/j.jpsychores.2008.10.006

Leventhal, M. A., Boyd, S., Moolchan, T. E., Waters, J. A., Lerman, C., \& Pickworth, W. (2007). Gender differences in acute tobacco withdrawal: effects on subjective, cognitive and physiological measures. Experimental and Clinical Psychopharmacology, 15(1), 21-36. doi:10.1037/1064-1297.15.1.21

Luk, J. W., \& Tsoh, J. Y. (2010). Moderation of gender on smoking and depression in Chinese Americans. Addictive Behaviors, 35(11), 1040-1043. doi: 10.1016/j.addbeh.2010.06.021

Morrell, H., Cohen, L. M., \& McChargue, D. E. (2010). Depression vulnerability predicts cigarette smoking among college students: gender and negative reinforcement expectancies as contributing factors. Addictive Behaviors, 35(6), 607-611. doi:10.1016/j. addbeh.2010.02.011

Nakajima, M., \& al'Absi, M. (2012). Predictors of risk for smoking relapse in men and women: a prospective examination. Psychology Addictive Behavior, 26(3), 633-637. doi:10.1037/a0027280

Oficina de las Naciones Unidas contra la Droga y el Delito. (2016). Guidelines on drug prevention and treatment for girls and women. Vienna: Autor. Recuperado https://www.unodc.org/documents/drug-prevention-and-treatment/unodc_2016_drug_ prevention_and_treatment_for_girls_and_women_E.pdf

Organización Mundial de la Salud. (2010). Gender, women, and the tobacco epidemic. Ginebra: Autor. Recuperado de http://apps. who.int/iris/bitstream/10665/44342/1/9789241599511_eng.pdf

Organización Mundial de la Salud. (2013). WHO report on the global tobacco epidemic. Ginebra: Autor. Recuperado de http://apps. who.int/iris/bitstream/10665/85380/1/9789241505871_eng.pdf? ua $=1$ [Accessed: 2018-01-17]

Organización Mundial de la Salud. (2017). WHO report on the global tobacco epidemic, 2017. Monitoring tobacco use and prevention policies. Ginebra: Autor. Recuperado de http://apps.who.int/iris/ bitstream/10665/255874/1/9789241512824-eng.pdf?ua=1

Pang, R. D., \& Leventhal, M. A. (2013). Sex differences in negative affect and lapse behavior during acute tobacco abstinence: a laboratory study. Experimental and Clinical Psychopharmacology, 21(4), 269-276. doi: 10.1037/a0033429

Pang, R. D., Zvolensky, J. M., Schmidt, B. N., \& Leventhal, M. A. (2015). Gender differences in negative reinforcement smoking expectancies. Nicotine \& Tobacco Research, 17(6), 750-754. doi:10.1093/ntr/ntu226
Perkins, A. K., Karelitz, L. J., Giedgowd, E. G., \& Conklin, A. C. (2013). Negative mood effects on craving to smoke in women versus men. Addictive Behaviors, 38(2), 1527-1531. doi: 10.1016/j. addbeh.2012.06.002

Pichon-Rivier, A., Bardach, A., Caporale, J., Alcaraz, A., Augustovski F., Caccavo, F., ... Hernández-San Román, E. (2013). Carga de enfermedad atribuible al tabaquismo en México. (Documento Técnico IECS Núm. 10). Buenos Aires, Argentina: Instituto de Efectividad Clínica y Sanitaria. Recuperado de http://media.controltabaco.mx/content/2013/Tabaquismo_en_México.pdf

Pozos-Radillo, B. E., Preciado-Serrano, M. L., Acosta-Fernández, M., Aguilera-Velasco, M. A., \& Delgado-García, D. D. (2014). Academic stress as a predictor of chronic stress in university students. Psicología Educativa, 20(1), 47-52. doi: 10.1016/j. pse.2014.05.006

Pozos-Radillo, B., Preciado-Serrano, M., Plascencia, A. R., Acosta-Fernández, M., \& Aguilera, M. (2015). Estrés académico y síntomas físicos, psicológicos y comportamentales en estudiantes mexicanos de una Universidad Pública. Ansiedad y Estrés, 21(1), 35-42.

Regueira, G., Suárez-Lugo, N., \& Jakimczuk, S. (2010). Estrategias para el control del tabaco con perspectiva de género en América Latina. Salud Pública de México, 52(2), S315-S320.

Reynales-Shigematsu, L. M., Zavala-Arciniega, L., Paz-Ballesteros, W. C., Gutiérrez-Torres, D. S., García-Buendía, J. C., Rodríguez-Andrade, M. A., ... Mendoza-Alvarado, L. (2017). Encuesta Nacional de Consumo de Drogas, Alcohol y Tabaco 2016-1017: Reporte de Tabaco. Ciudad de México, México: Instituto Nacional de Psiquiatría Ramón de la Fuente Muñiz, Instituto Nacional de Salud Pública, Comisión Nacional contra las Adicciones, Secretaría de Salud. Recuperado de https://drive.google.com/ file/d/1lktptvdu2nsrSpMBMT4FdqBIk8gikz7q/view

Robinson, S. M., Sobell, L. C., Sobell, M. B., \& Leo, G. I. (2014). Reliability of the timeline followback for cocaine, cannabis, and cigarette use. Psychology of Addictive Behaviors, 28(1), 154-162.

Robles, R., \& Páez, F. (2003). Estudio sobre la traducción al español y las propiedades psicométricas de las escalas de afectos positivo y negativo (PANAS). Salud Mental, 26(1), 69-75.

Robles, R., Varela, R., Jurado, S., \& Páez, F. (2001). Versión mexicana del inventario de ansiedad de Beck: propiedades psicométricas. Revista Mexicana de Psicología. 18(2), 211-218.

Roys, M., Weed, K., Carrigan, M., \& MacKillop, J. (2016). Associations between nicotine dependence, anhedonia, urgency and smoking motives. Addictive Behaviors, 62, 145-151. doi: 10.1016/j.addbeh.2016.06.002

Schleicher, H. E., Harris, K. J., Catley, D., \& Nazir, N. (2009). The role of depression and negative affect regulation expectancies in tobacco smoking among college students. Journal of American College Health, 57(5), 507-512. doi: 10.3200/JACH.57.5.507-512

Schmitz, M. J., Stotts, L. A., Mooney, E. M., DeLaune, A. K., \& MoeIler, F. G. (2007). Bupropion and cognitive-behavioral therapy for smoking cessation in women. Nicotine \& Tobacco Research, 9(6), 699-709. doi: 10.1080/14622200701365335 
Schnoll, A. R., Patterson, F., \& Lerman, C. (2007). Treating tobacco dependence in women. Journal of Womens Health, 16(8), 12111218. doi: 10.1089/jwh.2006.0281

Secretaría de Salud. (2012). Pautas para la prevención y la atención de las adicciones en universidades, institutos y escuelas de educación media superior y superior en México. México: Comisión Nacional contra las Adicciones.

Secretaría de Salud (2012). Perfil Epidemiológico del Tabaquismo en México. Recuperado de: http://www.epidemiologia.salud.gob.mx/ doctos/infoepid/publicaciones/2012/Monografias7_Tabaquismo_ Mex_julio12.pdf

Sobell, L. C., \& Sobell, M. B. (1980). Convergent validity: an approach to increasing confidence in treatment outcome conclusions with alcohol and drug abuses. En L. C. Sobell, M. B., Sobell, \& E. Ward (Eds.). Evaluating alcohol and drug abuse treatment effectiveness: recent advances (pp. 177-183). New York: Pergamon Press.

Suárez, N., \& Campos, E. (2010). Prevalencia y percepción del riesgo del tabaquismo en el área de salud del policlínico Dr. Jorge Ruiz Ramírez. Revista Cubana de Salud Pública, 36(2), 125-131.

Toribio-Ferrer, C., \& Franco-Bárcenas, S. (2016). Estrés académico: el enemigo silencioso del estudiante. Salud y Administración, 3(7), 11-18.

Torres, O. V., \& O'Dell, E. L. (2016). Stress is a principal factor that promotes tobacco use in females. Progress in Neuro-Psychopharmacology and Biological Psychiatry, 65(4), 260-268. doi: 10.1016/j. pnpbp.2015.04.005
Villatoro, J., Moreno, M., Oliva, N., Fregoso, D., Bustos, M., Fleiz. C., ... Medina-Mora, M. E. (2013). Consumo de alcohol, tabaco y otras drogas en la Ciudad de México. Medición 2012. México: Instituto Nacional de Psiquiatría Ramón de la Fuente Muñiz, Instituto para la Atención y la Prevención de las Adicciones, Administración Federal de los Servicios Educativos para el Distrito Federal.

Watson, D., Clark, L. A., \& Tellegen, A. (1988). Development and validation of brief measures of positive and negative affect: the PANAS scales. Journal of Personality and Social Psychology, 54(6), 1063-1070.

Weinberger, A. H., \& McKee, S. A. (2012). Gender differences in smoking following an implicit mood induction. Nicotine \& Tobacco Research, 14(5), 621-625. doi: 10.1093/ntr/ntr198

Wood, C. M., Cano-Vindel, A., Irruarrizaga, I., Dongil, E., \& Salguero, J. M. (2010). Relaciones entre estrés, tabaco y trastorno de pánico. Ansiedad y Estrés, 16(2-3), 309-325.

Xu, J., Azizian, A., Monterosso, J., Domier, C. P., Brody, A. L., Fong, T. W., ... London, E. D. (2008). Gender effects on mood and cigarette craving during early abstinence and resumption of smoking. Nicotine \& Tobacco Research, 10(11), 1653-1661. doi: $10.1080 / 14622200802412929$ 